PROCEEDINGS OF THE

AMERICAN MATHEMATICAL SOCIETY

Volume 135, Number 5, May 2007, Pages 1305-1308

S 0002-9939(06)08834-4

Article electronically published on November 15, 2006

\title{
ON THE COEFFICIENTS OF HILBERT QUASIPOLYNOMIALS
}

\author{
WINFRIED BRUNS AND BOGDAN ICHIM
}

(Communicated by Bernd Ulrich)

\begin{abstract}
The Hilbert function of a module over a positively graded algebra is of quasi-polynomial type (Hilbert-Serre). We derive an upper bound for its grade, i.e. the index from which on its coefficients are constant. As an application, we give a purely algebraic proof of an old combinatorial result (due to Ehrhart, McMullen and Stanley).
\end{abstract}

\section{Hilbert quASIPOLYNOMIALS}

Let $K$ be a field, and $R$ a positively graded $K$-algebra, that is, $R=\bigoplus_{i>0} R_{i}$ where $R_{0}=K$ and $R$ is finitely generated over $K$. We do not assume $R$ to be generated in degree 1 - the generators may be of arbitrarily high degree. The following theorem of Hilbert-Serre describes the Hilbert functions of finitely generated graded $R$-modules $M$.

Theorem 1. Let $M=\bigoplus_{i \in \mathbb{Z}} M_{i}$ be a finitely generated graded $R$-module of dimension $d, H\left(M,_{-}\right): \mathbb{Z} \rightarrow \mathbb{Z}$ the associated Hilbert function, and suppose that $r_{1}, \ldots, r_{d}$ is a homogeneous system of parameters for $M$.

Then there is a quasi-polynomial $Q_{M}$ of degree $d-1$, such that $H(M, n)=Q_{M}(n)$ for $n \gg 0$. Moreover, the period of $Q_{M}$ divides $\operatorname{lcm}\left(\operatorname{deg} r_{1}, \ldots, \operatorname{deg} r_{d}\right)$.

The terminology concerning quasipolynomials is explained as follows: a function $Q: \mathbb{Z} \rightarrow \mathbb{C}$ is called a quasipolynomial of degree $u$ if

$$
Q(n)=a_{u}(n) n^{u}+a_{u-1}(n) n^{u-1}+\ldots+a_{1}(n) n+a_{0}(n),
$$

where $a_{i}: \mathbb{Z} \rightarrow \mathbb{C}$ is a periodic function for $i=0, \ldots, u$, and $a_{u} \neq 0$. The period of $Q$ is the smallest positive integer $\pi$ such that

$$
a_{i}(n+m \pi)=a_{i}(n)
$$

for all $n, m \in \mathbb{Z}$ and $i=0, \ldots, u$.

For the reader's convenience, we include a short proof the Hilbert-Serre theorem, or rather its reduction to the classical theorem of Hilbert. By definition of homogeneous system of parameters, $M$ is a finitely generated module over $K\left[r_{1}, \ldots, r_{d}\right]$ (which is isomorphic to a polynomial ring over $K$ ). Therefore we may assume that $R=K\left[r_{1}, \ldots, r_{d}\right]$. Let $S$ be the subalgebra of $R$ generated by its homogeneous elements of degree $p=\operatorname{lcm}\left(\operatorname{deg} r_{1}, \ldots, \operatorname{deg} r_{d}\right)$. Then it is not hard to see that $R$ is a finitely generated $S$-module. Therefore $M$ is a finitely generated $S$-module,

Received by the editors December 22, 2005.

2000 Mathematics Subject Classification. Primary 13A02.

Key words and phrases. Hilbert quasi-polynomial, Ehrhart quasi-polynomial.

(C)2006 American Mathematical Society 
too, and $\operatorname{dim}_{S} M=\operatorname{dim}_{R} M$. As a last reduction step, we can replace $R$ by $S$ and assume that $R$ is generated by its elements of degree $p$.

Then we have the decomposition

$$
M=M^{0} \oplus \ldots \oplus M^{p-1}, \quad M^{k}=\bigoplus_{i \equiv k(p)} M_{i},
$$

into finitely generated $R$-modules, and $\operatorname{dim} M=\max _{k} \operatorname{dim} M^{k}$.

Let us consider a single module $M^{k}$. Then we can normalize the degrees in $R$ dividing them by $p$ and re-grade $M^{k}$ by giving degree $(i-k) / p$ to the elements of its degree $i$ component in the original grading, $i \equiv k(p)$. By Hilbert's theorem, the Hilbert function of $M^{k}$ re-graded is given by a true polynomial $P_{k}(n)$ for $n \gg 0$.

Returning to $M$ we obtain

$$
H(M, n)=P_{k}((n-k) / p), \quad n \equiv k(p), n \gg 0,
$$

and this proves the theorem.

It is clear that any improvement of the theorem depends on the "coherence" of the modules $M^{k}$. The reduction in the proof above forgets the original module structure to a large extent. Clearly, in the extreme case in which $R$ is generated by its degree $p$ elements, $M$ is just a direct sum of the independent modules $M^{k}$. But if the $M^{k}$ are sufficiently related, then one can say more on $Q_{M}$.

\section{The grade of Hilbert quasipolynomials}

It is a natural question to ask how close $Q_{M}$ is to being a true polynomial. The next theorem, which is the main result of this paper, provides an answer. Following Ehrhart [E], we let the grade of $Q$ denote the smallest integer $\delta \geq-1$ such that $a_{i}\left({ }_{-}\right)$is constant for all $i>\delta$.

Theorem 2. Let $M=\bigoplus_{i \in \mathbb{Z}} M_{i}$ be a finitely generated graded $R$-module of dimension $d$, and

$$
Q(n)=a_{d-1}(n) n^{d-1}+a_{d-2}(n) n^{d-2}+\ldots+a_{1}(n) n+a_{0}(n)
$$

its Hilbert quasi-polynomial with period $\pi$. Let $I$ be the ideal of $R$ generated by all homogeneous elements $x$ of $R$ such that $\operatorname{gcd}(\operatorname{deg} x, \pi)=1$. Then

$$
\text { grade } Q<\operatorname{dim} M / I M \text {. }
$$

The theorem will be proved by an induction based on the following lemma, in which, as usual, $(0: x)_{M}=\{u \in M: x u=0\}$.

Lemma 3. With the notation of the theorem, if $\operatorname{dim} M / I M<\operatorname{dim} M$, then there is a homogeneous $x \in I$ with $\operatorname{gcd}(\operatorname{deg} x, \pi)=1$, such that

(a) $\operatorname{dim} M / x M=\operatorname{dim} M-1$,

(b) $\operatorname{dim}(0: x)_{M} \leq \operatorname{dim} M-1$.

Proof. Let $D(M)=\{\mathfrak{p} \in V(M), \operatorname{dim} A / \mathfrak{p}=\operatorname{dim} M\}=\left\{\mathfrak{p}_{1}, \ldots, \mathfrak{p}_{r}\right\}$. Clearly $I \not \subset \mathfrak{p}_{i}$ for $i=1, \ldots, r$. By prime avoidance, we conclude that $I \not \subset \mathfrak{p}_{1} \cup \ldots \cup \mathfrak{p}_{r}$. By induction on $r$, we show that

$$
S=\{x \in I, x \text { homogeneous, } \operatorname{gcd}(\operatorname{deg} x, \pi)=1\} \not \subset \bigcup_{i=1}^{r} \mathfrak{p}_{i} .
$$


This is clear for $r=1$. For $1 \leq j \leq r$, we may assume by induction that

$$
S \not \subset \bigcup_{i=1, i \neq j}^{r} \mathfrak{p}_{i}
$$

Assume that $S \subset \mathfrak{p}_{1} \cup \cdots \cup \mathfrak{p}_{r}$. Then for each $j=1, \ldots, r$ there is $x_{j} \in S$ such that

$$
x_{j} \in \mathfrak{p}_{j} \backslash\left(\bigcup_{i=1, i \neq j}^{r} \mathfrak{p}_{i}\right) .
$$

Let $\operatorname{deg} x_{1}=\alpha$ and $\operatorname{deg} x_{2} \ldots x_{r}=\beta$. Then $x=x_{1}^{\operatorname{lcm}(\alpha, \beta) / \alpha}+\left(x_{2} \ldots x_{r}\right)^{\operatorname{lcm}(\alpha, \beta) / \beta} \in$ $S$, since it is homogeneous, and $\operatorname{gcd}(\operatorname{lcm}(\alpha, \beta), \pi)=1$. Now

$$
x_{1} \in \mathfrak{p}_{1} \backslash\left(\bigcup_{i=2}^{r} \mathfrak{p}_{i}\right) \quad \text { and } \quad x_{2} \ldots x_{r} \in\left(\bigcap_{i=2}^{r} \mathfrak{p}_{i}\right) \backslash \mathfrak{p}_{1} \quad \text { implies } \quad x \notin \bigcup_{i=1}^{r} \mathfrak{p}_{i}
$$

a contradiction.

Let $x \in S \backslash\left(\mathfrak{p}_{1} \cup \ldots \cup \mathfrak{p}_{r}\right)$. Then $\operatorname{dim} M / x M=\operatorname{dim} M-1$. Moreover every prime ideal in the support of $(0: x)_{M}$ is in the support of $M / x M$. Thus $\operatorname{dim}(0: x)_{M} \leq$ $\operatorname{dim} M-1$.

Proof of Theorem 2. We prove by induction on $\operatorname{dim} M=d$ that $\operatorname{dim} M / I M \leq \gamma$ implies $a_{j}(-)$ constant for all $j \geq \gamma$. This is clear if $d \leq \gamma$ (then $j \geq \gamma$ implies $a_{j}(\mathbf{-})=0$ ), so we may assume $d>\gamma$. Let $x$ be as in the lemma, and $g=\operatorname{deg} x$.

Set $M^{\prime}=M / x M$ and $M^{\prime \prime}=(0: x)_{M}$. Then $M^{\prime} / I M^{\prime} \cong M / I M$ and certainly $\operatorname{dim} M^{\prime \prime} / I M^{\prime \prime} \leq \gamma$. Since $\operatorname{dim} M^{\prime}, \operatorname{dim} M^{\prime \prime}<\operatorname{dim} M$, we may assume by induction that $H(M / x M, n)$ and $H\left((0: x)_{M}, n\right), n \gg 0$, are quasipolynomials of grade $<\gamma$.

The exact sequence

$$
0 \longrightarrow(0: x)_{M}(-g) \longrightarrow M(-g) \stackrel{x}{\longrightarrow} M \longrightarrow M / x M \longrightarrow 0
$$

gives the equation

$$
H(M, n)-H(M, n-g)=H(M / x M, n)-H\left((0: x)_{M}, n-g\right) .
$$

For a quasipolynomial $Q$ it is easy to see that $Q(n-g)$ has the same grade as $Q$. Therefore the right-hand side in the previous equation is a quasipolynomial of grade $<\gamma$ for $n \gg 0$, and so this holds for the left-hand side, too. So it remains only to apply the following lemma.

Lemma 4. Let $Q(n)=\sum a_{k}(n) n^{k}$ be a quasipolynomial. If $Q(n)-Q(n-g)$ is of grade $<\gamma$ for some $g$ coprime to the period $\pi$ of $Q$, then grade $Q<\gamma$.

Proof. Let $u=\operatorname{deg} Q$ and let us first compare the leading coefficients. We can assume $\gamma \leq u$. Then one has $a_{u}(n)-a_{u}(n-g)=C$ for some constant $C$ and all $n$, and so $a_{u}(n)-a_{u}(n-\pi g)=\pi C$. Since $\pi$ is the period, we conclude that $C=0$, and $a_{u}(n)=a_{u}(n-g)$. But $g$ is coprime to $\pi$, and it follows that $a_{u}$ is constant.

The descending induction being started, one argues as follows for the lower coefficients. Suppose that $k \geq \gamma$. Then $a_{k}(n)-a_{k}(n-g)$ is a polynomial in the coefficients $a_{j}$ for $j>k$ and $g$. Since the higher coefficients are constant by induction, it follows that $a_{k}(n)-a_{k}(n-g)$ is constant, too, and the rest of the argument is as above. 


\section{An APPLICATION TO RATIONAL POLYTOPES}

In this section we shall give a purely algebraic proof of an old theorem, which was conjectured by Ehrhart ([E], p. 53), and proved independently by McMullen (see $[\mathrm{M}]$ ) and Stanley $(\underline{\mathrm{S}}$, Theorem 2.8).

Theorem 5. Let $P$ be a d-dimensional rational convex polytope in $\mathbb{R}^{m}$, and let the Ehrhart quasi-polynomial of $P$ be

$$
E_{P}(n)=a_{d}(n) n^{d}+a_{d-1}(n) n^{d-1}+\ldots+a_{1}(n) n+a_{0}(n) .
$$

Suppose that for some $\delta$ the affine span of every $\delta$-dimensional face of $P$ contains a point with integer coordinates. Then grade $E_{P}<\delta$.

Proof. We choose a field $K$ and let $R$ be the Ehrhart ring of $P$. It is the vector subspace of $K\left[X_{1}^{ \pm 1}, \ldots, X_{m}^{ \pm 1}, T\right]$ spanned by all Laurent monomials $X^{a} T^{n}=$ $X_{1}^{a_{1}} \ldots X_{m}^{a_{m}} T^{n}$ where $a=\left(a_{1}, \ldots, a_{m}\right) \in n P, n \in \mathbb{Z}, n \geq 0$. By Gordan's lemma it follows easily that $R$ is a finitely generated, positively graded $K$-algebra, where we use the exponent of $T$ as the degree of a monomial. The Ehrhart function of $P$ is just the Hilbert function of $R$. (See Chapter 6 of $[\mathrm{BH}]$ for more information.)

Let $\pi$ be the period of $E_{P}$, and $F$ a $\delta$-dimensional face of $P$. Since the affine span of $F$ contains a point with integer coordinates, $n F$ contains a point with integer coordinates for all $n \gg 0$. We chose $n$ big enough so that $n F$ contains a point $m_{F}$ with integer coordinates for every $\delta$-dimensional face $F$, and $\operatorname{gcd}(n, \pi)=1$.

Now let $J \subset R$ be the ideal generated by the monomials $X^{m_{F}} T^{n}$. If $\operatorname{dim} R / J \leq \delta$, then we are done by Theorem 2 because the ideal $I$ in Theorem 2 contains $J$.

Since $J$ is a monomial ideal, $\operatorname{Ass}_{R} R / J$ consists of monomial prime ideals. In particular, $\operatorname{Min}_{R} R / J$ consists of monomial prime ideals. By theorem 6.1.7 of [BH], for each $\mathfrak{p} \in \operatorname{Min}_{R} R / J$, there is a face $G_{\mathfrak{p}}$ of $P$, such that $\mathfrak{p}$ is generated by all monomials outside the cone associated with $G_{\mathfrak{p}}$. One has $\operatorname{dim} R / \mathfrak{p}=\operatorname{dim} G_{\mathfrak{p}}+1$. Since $J \subset \mathfrak{p}$, it follows that $\operatorname{dim} G_{\mathfrak{p}} \leq \delta-1$. So $\operatorname{dim} R / J=\max \{\operatorname{dim} R / \mathfrak{p}, \mathfrak{p} \in$ $\left.\operatorname{Min}_{R} R / J\right\} \leq(\delta-1)+1=\delta$.

\section{REFERENCES}

[BH] W. Bruns and J. Herzog. Cohen-Macaulay Rings. Rev. ed. Cambridge University Press, 1998.

[E] E. Ehrhart. Polynômes arithmétiques et Méthode des Polyèdres en Combinatoire. International Series of Numerical Mathematics. 35, Birkhäuser Verlag, 1977. MR0432556 (55:5544)

[M] P. McMullen. Lattice invariant valuations on rational polytopes. Arch. Math. 31 (1978/79), 509-516. MR0526617 (80d:52011)

[S] R. Stanley. Decompositions of rational convex polytopes. Ann. Discr. Math. 6 (1980), 333342. MR0593545 (82a:52007)

FB Mathematik/Informatik, Universität Osnabrück, 49069 Osnabrück, Germany

E-mail address: winfried@math.uos.de

FB Mathematik/Informatik, Universität Osnabrück, 49069 Osnabrück, Germany; And Institute of Mathematics, C.P. 1-764, 70700 Bucharest, Romania

E-mail address: bogdan.ichim@math.uos.de; bogdan.ichim@imar.ro 